\title{
Electromagnetic Device Design Based on RBF Models and Two New Sequential Optimization Strategies
}

\author{
Gang Lei ${ }^{1}$, G. Y. Yang ${ }^{1}$, K. R. Shao ${ }^{1}$, Youguang Guo ${ }^{2}$, Jianguo Zhu ${ }^{2}$, and J. D. Lavers ${ }^{3}$, Fellow, IEEE \\ ${ }^{1}$ College of Electrical and Electronic Engineering, Huazhong University of Science and Technology, Wuhan 430074, China \\ ${ }^{2}$ Faculty of Engineering, University of Technology, Sydney, N.S.W. 2007, Australia \\ ${ }^{3}$ Department of Electrical and Computer Engineering, University of Toronto, Toronto, ON M5S 3G4, Canada
}

\begin{abstract}
We present two new strategies for sequential optimization method (SOM) to deal with the optimization design problems of electromagnetic devices. One is a new space reduction strategy; the other is model selection strategy. Meanwhile, radial basis function (RBF) and compactly supported RBF models are investigated to extend the applied model types for SOM. Thereafter, Monte Carlo method is employed to demonstrate the efficiency and superiority of the new space reduction strategy. Five commonly used approximate models are considered for the discussion of model selection strategy. Furthermore, by two TEAM benchmark examples, we can see that SOM with the proposed new strategies and models can significantly speed the optimization design process, and the efficiency of SOM depends a little on the types of approximate models.
\end{abstract}

Index Terms-Approximate models, electromagnetic device, Monte Carlo method, optimization methods, radial basis function (RBF).

\section{INTRODUCTION}

A PPROXIMATE models have been widely employed as the surrogates of physical models (e.g., finite element model) in the optimization design of electromagnetic devices. The main reason is that the computation cost of direct optimization of a physical model is always very expensive. So many kinds of approximate models have been investigated in this field [1]. The optimization processes based on these models are always proved fast. However, the efficiency of this method highly depends on the experiment design of modeling process, such as sampling methods and points. Therefore, the accuracy of this method is still needed to be improved.

To improve the optimization efficiency, we have introduced sequential optimization method (SOM) to solve such design problems [2], [3]. Unlike the traditional methods, SOM can optimize the approximate models and algorithms in one optimization process. It has a good performance in the practical applications. However, SOM was discussed only for response surface model and Kriging model in the former study, so two kinds of radial basis function (RBF) models are first presented in this work. Meanwhile, given variety of models, it is still a problem to select the most appropriate model for the device under study. That is to say, we need to discuss the model selection strategy for SOM. Furthermore, to improve the optimization efficiency of SOM and to make full use of the sampled finite element points, a new and important space reduction strategy is presented in this work.

\section{RBF APPROXIMATE MODELS}

RBF model is a determinate parametric model. It can rapidly replace the finite element model by using a linear combination

Manuscript received December 03, 2009; accepted February 13, 2010. Current version published July 21, 2010. Corresponding author: G. Lei (e-mail: gangleimc@gmail.com).

Color versions of one or more of the figures in this paper are available online at http://ieeexplore.ieee.org.

Digital Object Identifier 10.1109/TMAG.2010.2043717 of radial symmetric functions to interpolate sample data and reconstruct the response surface.

Given $n$ sample points $\left\{x_{1}, x_{2}, \ldots, x_{n}\right\}$ and their responses, for an input $x$, the response value of RBF model is given by

$$
\hat{y}(x)=\sum_{i=1}^{n} \beta_{i} \cdot \varphi\left(\left\|x-x_{i}\right\|\right)
$$

where $\beta_{i}$ are model parameters, $\varphi(r)$ is $\mathrm{RBF}$, and $\|\cdot\|$ is the Euclidean norm. Gauss and multiquadric (MQ) RBF are the two most commonly used RBF, which are also considered in this work. They have the forms as

$$
\begin{aligned}
\text { Gauss }: \varphi(r) & =\exp \left(-c^{2} r^{2}\right) \\
\text { MQ }: \varphi(r) & =\left(r^{2}+c^{2}\right)^{1 / 2}
\end{aligned}
$$

where $c$ is a shape parameter. RBF model can effectively supersede the finite element simulation for objective functions and conditions. However, it is generally globally supported and "poorly conditioned," especially when the number of sampling points increases significantly [4].

Compactly supported RBF (CSRBF) model is a promising improvement in this aspect. The improvement of CSRBF lies in the basis function, which is compactly supported and positive-definite compared to that in RBF. When this model is used, the evaluation of (1) will not run over the whole set of the sampled points. It only includes the points in the compactly supported domain, and then the coefficient matrix of model equations will be sparse. A series of positive-definite CSRBF have been developed [4]. The following two CSRBF are studied in this work:

$$
\begin{aligned}
\text { CSRBF1 }: \varphi(r)= & (1-r)_{+}^{6}(6+36 r \\
& \left.+82 r^{2}+72 r^{3}+30 r^{4}+5 r^{5}\right) \\
\text { CSRBF2 }: \varphi(r)= & (1-r)_{+}^{8}\left(1+8 r+25 r^{2}+32 r^{3}\right)
\end{aligned}
$$

where $r$ is a norm with respect to the radius of the compactly supported domain. $(1-r)_{+}$is a truncated function. If $r$ ranges from 0 to 1 , its value is $1-r$; otherwise it is 0 . 


\section{NEW SEQUENTIAL OPTIMIZATION STRATEGY}

SOM has been successfully employed to solve the electromagnetic design problems. It is composed of coarse optimization process and fine optimization process. The main purpose of the former is to reduce the design space [2]. Space reduction strategy plays an important role in this process. It has a great effect on the efficiency of SOM.

The design purpose of the former space reduction strategy is to minimize the distance between the mean of next design range and the current optimal result [2], [3]. It is accurate for the distance minimization, but it has not considered how to effectively save the number of sampling points. To make the most of the sample points that are sampled in the last set, we present a new space reduction strategy in this work.

Suppose $x^{(k)}=\left[x_{l i}^{(k)}, x_{u i}^{(k)}\right]$ is boundary of the $i$ th variable in the $k$ th optimization process, $i=1,2, \ldots, D . l^{(k)}$ is interval, $h^{(k)}$ is step size, $N^{(k)}$ is the number of sample points, and $\mathrm{S}^{(k)}$ is sample set. $x_{o}^{(k)}$ and $f^{(k)}$ are the optimal result and corresponding function value, respectively. The new space reduction strategy is designed with the following two steps.

Reduction step:

$$
\begin{aligned}
& \hat{x}_{l i}^{(k+1)}=\max \left\{x_{l i}^{(k)}, \operatorname{round}\left[\left(x_{o i}^{(k)}-\Delta l\right) / \Delta h\right] \Delta h\right\} \\
& \hat{x}_{u i}^{(k+1)}=\min \left\{x_{u i}^{(k)}, \operatorname{round}\left[\left(x_{o i}^{(k)}+\Delta l\right) / \Delta h\right] \Delta h\right\} .
\end{aligned}
$$

Correction step:

$$
\begin{aligned}
& x_{l i}^{(k+1)}=x_{l i}^{(k)}+\operatorname{round}\left[2\left(\hat{x}_{l i}^{(k+1)}-x_{l i}^{(k)}\right) / h_{i}^{(k)}\right] \cdot h_{i}^{(k)} / 2 \\
& x_{u i}^{(k+1)}=x_{l i}^{(k)}+\operatorname{round}\left[2\left(\hat{x}_{u i}^{(k+1)}-x_{l i}^{(k)}\right) / h_{i}^{(k)}\right] \cdot h_{i}^{(k)} / 2 .
\end{aligned}
$$

In the above, function $\operatorname{round}(x)$ is rounded to the nearest integer of $x . \Delta l=l_{i}^{(k)} / n_{l}$ and $\Delta h=h_{i}^{(k)} / n_{h}$, where $n_{l}$ and $n_{h}$ are the reduction factors. From the former study, $n_{l}=2$ and $n_{h}=8$ are suitable for most cases [3]. Now, we give a comparison about the efficiency between the former and new space reduction strategies.

For example, suppose initialization design space is $[0,1]$, $N$ is 6, and uniform sampling method is used. Then, the first sample data $S^{(1)}=\{0.0,0.2,0.4,0.6,0.8,1.0\}$. Suppose the optimal value is 0.35 . From the former space reduction strategy, the next design space is $[0.1,0.6]$, and the next sample set $\mathrm{S}^{(2)}=\{0.1,0.2,0.3,0.4,0.5,0.6\}$. Obviously, three sample points have been sampled in $\mathrm{S}^{(1)}$. In other words, $50 \%$ computation cost is saved. Now, if we use new space reduction strategy, the next sample space is $[0.2,0.6]$, and $\mathrm{S}^{(2)}=\{0.2,0.3,0.4,0.5,0.6\}$. Obviously, $60 \%$ computation cost is saved.

As another example, suppose the optimal value is 0.3; from the former strategy, the next sample space is [0.05, $0.55]$, and $\mathrm{S}^{(2)}=\{0.05,0.15,0.25,0.35,0.45,0.55\}$. Obviously, no sample points have been sampled. If we use the new strategy, the next sample space is $[0.0,0.6]$, and $\mathrm{S}^{(2)}=\{0.0,0.1,0.2,0.3,0.4,0.5,0.6\}$. Obviously, four sample points have been sampled in the $S^{(1)}$. In other words,
TABLE I

MeAn SAVING RATES by Two StRategies

\begin{tabular}{|c|c|c|c|c|c|}
\hline$N$ & 2 & 3 & 4 & 5 & 6 \\
\hline Former & 0.3123 & 0.2293 & 0.2531 & 0.2428 & 0.2375 \\
\hline New & 0.5312 & 0.5415 & 0.5383 & 0.5334 & 0.5221 \\
\hline
\end{tabular}

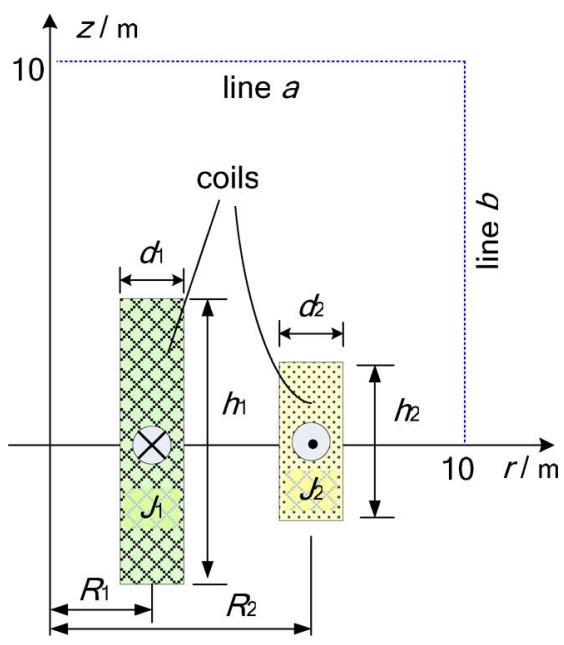

Fig. 1. Geometry configuration of SMES.

$57.14 \%$ computation cost is reduced. Thus, the new strategy is more reasonable.

Table I shows mean saving rates of sample points about the former and new space reduction strategies with Monte Carlo method. For each strategy and every sample number $N, 10^{6}$ random numbers are generated as the current optimal points by Monte Carlo method. Then, we can get the mean saving rate for each case. From Table I, we can see that all saving rates by the new strategy are more than $50 \%$, which are obviously higher than those by the former strategy.

To sum up, the starting point of the new space reduction strategy is clearly different from the former one. The former focused on the distance minimization, while the sampled points can be fully utilized in the next modeling process by the new strategy. Therefore, the cost of finite element analysis can be saved to a great extent.

\section{EXPERIMENTS}

\section{A. TEAM Workshop Problem 22}

It is a benchmark problem for the optimization design of superconducting magnetic energy storage (SMES) [1]-[3], [5], [6]. Fig. 1 shows the design model. There are many ways to define the objective function for this problem. In this work, it is defined as $f(x)=B_{\text {stray }} / B_{\text {norm }}$, where $B_{\text {stray }}$ is mean stray fields on lines $a$ and $b$, and $B_{\text {norm }}$ is $3 \mathrm{mT}$. Three constraints are: $h(x)=|E / 180-1|=0, q(x)=R_{1}+d_{1} / 2+d_{2} / 2-R_{2}<0$, and $g(x)=\left|B_{\max }\right|-\min \left[\left(54-\left|J_{i}\right|\right) / 6.4\right] \leq 0$, for $i=1,2$.

There are two cases about this problem, namely discrete case (three-parameters problem) and continuous case (eight-parameters problem). In the former case, only the dimensions of the 


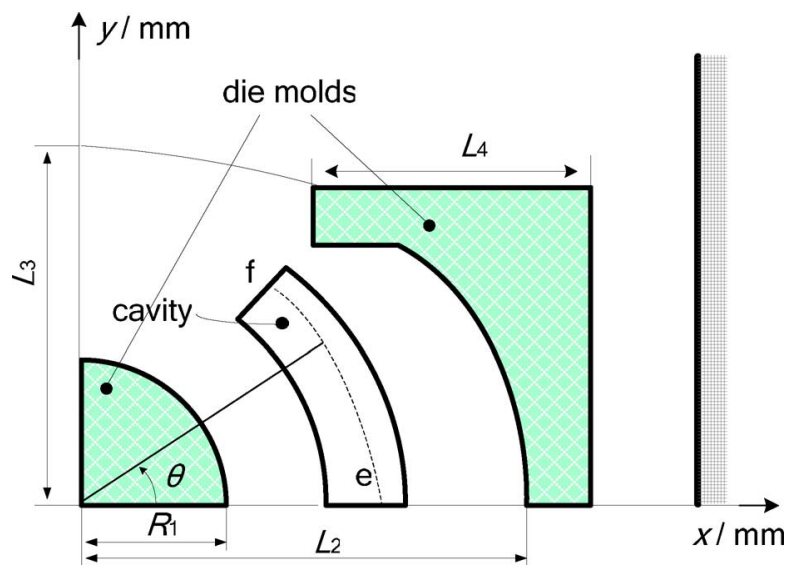

Fig. 2. Central configuration of die press model.

outer coil $\left(R_{2}, h_{2}, d_{2}\right)$ are the optimization parameters. Moreover, it should be noted that the second constraint is only defined for the continuous case, and it is only used in the algorithm sampling process. Thus, the final objective function for two cases is the same, and it has the form as

$$
F(x)=f(x)+1000\left[h(x)^{2}+\max (g(x), 0)^{2}\right] .
$$

\section{B. TEAM Workshop Problem 25}

It is also a benchmark problem for the optimization of die press model [3], [7], [8]. Fig. 2 is the central geometry configuration of the model. Four parameters should be optimized to ensure that the radial magnetic induction on line e-f equals $0.35 \mathrm{~T}$. The objective function is

$$
W(x)=\sum_{i=1}^{10}\left[\left(B_{x i \mathrm{p}}-B_{x i \mathrm{o}}\right)^{2}+\left(B_{y i \mathrm{p}}-B_{y i \mathrm{o}}\right)^{2}\right]
$$

where subscripts $\mathrm{p}$ and $\mathrm{o}$ mean the calculated and specified values, respectively. This problem also has a three-parameters problem. In this case, $L_{3}$ is fixed at $14 \mathrm{~mm}$.

\section{DISCUSSIONS AND RESUlTS}

In the following design examples, differential evolution algorithm (DEA) is used as the optimization algorithm [9]. The parameters of SOM and DEA used in this work are the same as the former study [3]. It should be pointed out that the average convergence time of one SOM optimization loop process is less than the time of a finite element point sampling process for two problems. Thus, the time for algorithm optimization process can be neglected compared to that of sampling process. That is to say, we only need to compare the computation costs of finite element analysis for different methods in the following discussion. The determination strategy of $N^{(1)}$ for the two problems in this work is defined as follows. If $l^{(1)} \leq 0.4$, its value is 3 ; if $l^{(1)} \geq 0.8$, its value is 5 ; for else cases, it is 4 .

\section{A. Discrete Case of SMES}

Table II lists optimization results of this case. Four main conclusions can be drawn from the table.

1) For the direct optimization with DEA, 2310 finite element sample points (FESP) are needed to get the optimal result, and function value is 0.343 .

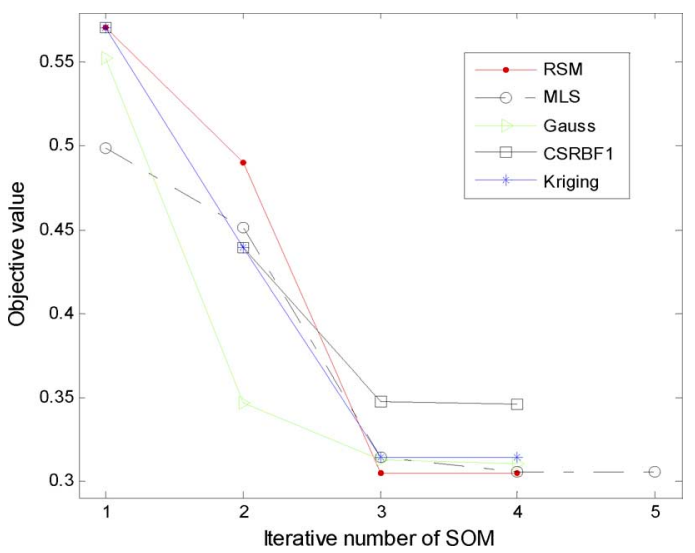

Fig. 3. Convergence history of SOM with different models.

TABLE II

OPTIMIZATION RESULTS OF DISCRETE CASE OF SMES

\begin{tabular}{|c|c|c|c|c|c|c|}
\hline Var. & Unit & DEA & Gauss & MQ & CSRBF1 & CSRBF2 \\
\hline$R_{2}$ & $\mathrm{~m}$ & 3.18 & 3.12 & 3.07 & 3.06 & 3.03 \\
\hline$h_{2} / 2$ & $\mathrm{~m}$ & 0.428 & 0.309 & 0.295 & 0.302 & 0.316 \\
\hline$d_{2}$ & $\mathrm{~m}$ & 0.211 & 0.295 & 0.328 & 0.325 & 0.325 \\
\hline $\mathrm{B}_{\text {stray }}$ & $\mathrm{mT}$ & 1.03 & 0.93 & 0.97 & 1.01 & 1.23 \\
\hline $\mathrm{E}$ & $\mathrm{MJ}$ & 180.00 & 179.94 & 179.64 & 179.45 & 179.24 \\
\hline $\mathrm{F}$ & - & 0.343 & 0.310 & 0.327 & 0.346 & 0.428 \\
\hline FESP & - & 2310 & 214 & 129 & 122 & 122 \\
\hline
\end{tabular}

2) For SOM with two types of RBF models, the result given by Gauss RBF model is better than that by MQ RBF model. Both of them are better than that of DEA.

3) For SOM with two types of CSRBF models, the result given by the CSRBF1 is better than that of CSRBF2. However, both of them are worse than those of RBF models and DEA.

4) The result given by Gauss RBF model is the best one in the listed results. The FESP of CSRBF1 is the least one in the listed results. Only 122 FESP are sampled for that case, which is $57.01 \%$ compared to that of Gauss RBF model. Furthermore, all the FESP needed by RBF and CSRBF models are less than 1/10 compared to that given by DEA. As Gauss RBF and CSRBF1 are the better ones in each type of model, we only consider them in the latter discussion.

Now, we use this problem to discuss the model selection strategy for SOM. Five types of models are considered here: response surface model (RSM), moving least square (MLS), Gauss RBF, CSRBF1, and Kriging models. They are the five most widely used approximate models for electromagnetic design problems.

The convergence history of SOM with different models can be seen in Fig. 3. Here, iterative number of SOM means the optimization loop number of coarse and fine optimization processes. From the figure, we can see that all the results have no distinct differences, so the type of approximate model has little effect on the efficiency of SOM for this problem.

As we have pointed out, CSRBF is an improvement of RBF in the theoretic analysis. However, from Table II and Fig. 3, we can 
TABLE III

OPTIMIZATION RESUlTS OF CONTINUOUS CASE OF SMES

\begin{tabular}{|c|c|c|c|c|}
\hline Var. & Unit & DEA & Gauss & CSRBF1 \\
\hline $\mathrm{R}_{1}$ & $\mathrm{~m}$ & 2.3816 & 1.2407 & 1.2407 \\
\hline $\mathrm{R}_{2}$ & $\mathrm{~m}$ & 3.3772 & 3.1900 & 3.1904 \\
\hline $\mathrm{h}_{1} / 2$ & $\mathrm{~m}$ & 1.1182 & 1.1312 & 1.1312 \\
\hline $\mathrm{h}_{2} / 2$ & $\mathrm{~m}$ & 0.3659 & 0.2068 & 0.2109 \\
\hline $\mathrm{d}_{1}$ & $\mathrm{~m}$ & 0.1884 & 0.6416 & 0.6416 \\
\hline $\mathrm{d}_{2}$ & $\mathrm{~m}$ & 0.6531 & 0.5594 & 0.5470 \\
\hline $\mathrm{J}_{1}$ & $\mathrm{MA} / \mathrm{m}^{2}$ & 22.5717 & 10.6325 & 10.6325 \\
\hline $\mathrm{J}_{2}$ & $\mathrm{MA} / \mathrm{m}^{2}$ & -11.0582 & -13.9904 & -13.9904 \\
\hline $\mathrm{B}_{\text {stray }}$ & $\mathrm{mT}$ & 2.2725 & 2.8553 & 2.8301 \\
\hline $\mathrm{E}$ & $\mathrm{MJ}$ & 178.75 & 179.05 & 179.00 \\
\hline $\mathrm{F}$ & - & 0.8057 & 0.9796 & 0.9742 \\
\hline $\mathrm{FESP}$ & - & 4720 & 1424 & 1404 \\
\hline
\end{tabular}

see that the efficiencies of two CSRBF models are lower than the other models for this problem, including two RBF models. The reason is that the sample points in SOM are very small, and the "poorly conditioned" of RBF will not appear. Actually, the efficiency of RBF models is higher than CSRBF models for $\mathrm{SOM}$. This conclusion can be also affirmed by the following two examples.

\section{B. Three-Parameters Case of TEAM Workshop Problem 25}

From the analysis, we can get the following conclusions.

1) For the direct optimization of DEA, 2420 FESP to get the optimal result, which is [7.555, 14.721, 14.867], and the square error is $4.14 * 10^{-4}$.

2) For SOM with Gauss RBF and CSRBF1 models, the results are the same; it is [7.613, 14.625, 15.719]. Only 328 FESP are needed, which is $13.55 \%$ compared to that of DEA. The square error is $1.49 * 10^{-3}$, which is a little bigger than that of DEA, but also satisfies the design specifications.

3) For the model selection strategy about this problem, from the similar analysis, we can also get that model selection has very little effect on the results of SOM.

\section{Continuous Case of SMES}

For this case, we first use dimension reduction optimization method to convert it into a low-dimensional problem [3]. Then, new SOM strategy is used to educe the results. Table III shows the optimal solutions. Three main conclusions can be drawn from the table.

1) For DEA to get the optimal solution, 4720 FESP are needed, and objective function value is 0.8057 .

2) For the SOM with Gauss RBF model, only 1424 FESP (224 from SOM) are needed. It is about $30.17 \%$ compared to that of DEA. The objective function value is a little bigger than that of DEA. However, the error of $E$ is
$0.95 \mathrm{MJ}$ (or $0.53 \%$ ), which is smaller than that given by DEA (1.25 MJ).

3) For the optimization with CSRBF1 model, the needed FESP are even less than that of Gauss RBF model. Its objective function value is also a little bigger than that of DEA, while the error of energy is $1 \mathrm{MJ}$, which is smaller than that given by DEA. Thus, the finite-element computational efforts can be significantly reduced by the proposed methods. If we increase the sample points in the dimension reduction optimization process, we can get a better solution. Moreover, the dimensions of the inner coil are obviously different. To get a solution with higher stability, we may need some other constraints, such as minimizing the volume.

\section{CONCLUSION}

In summary, several types of RBF and CSRBF models are introduced to extend applied model types of SOM. The new space reduction strategy can increase the saving rate of sample points. From the examples, we can see that SOM with them can produce satisfactory solutions, and can comparably decrease the total cost. Furthermore, the efficiency of SOM depends a little on the model types.

\section{ACKNOWLEDGMENT}

This work was supported by the NSFC under Grant 50877029 and by the China Postdoctoral Science Foundation under Grant 20090460929.

\section{REFERENCES}

[1] L. D. Wang and D. A. Lowther, "Selection of approximation models for electromagnetic device optimization," IEEE Trans. Magn., vol. 42, no. 2, pp. 1227-1230, Feb. 2006.

[2] G. Lei, K. R. Shao, Y. Guo, J. Zhu, and J. D. Lavers, "Sequential optimization method for the design of electromagnetic device," IEEE Trans. Magn., vol. 44, no. 11, pp. 3217-3220, Nov. 2008.

[3] G. Lei, K. R. Shao, Y. Guo, J. Zhu, and J. D. Lavers, "Improved sequential optimization method for high dimensional electromagnetic optimization problems," IEEE Trans. Magn., vol. 45, no. 10, pp. 3993-3996, Oct. 2009.

[4] K. Z. Song, X. Zhang, and M. W. Lu, "Meshless method based on collocation with consistent compactly supported radial basis functions," Acta Mechanica Sinica, vol. 20, no. 5, pp. 551-557, 2004.

[5] "TEAM workshop problem 22-SMES optimisation benchmark," 1996 [Online]. Available: http://www.igte.tugraz.at/archive/team/ index.htm

[6] F. Campelo, F. G. Guimaraes, H. Igarashi, and J. A. Ramirez, "A clonal selection algorithm for optimization in electromagnetics," IEEE Trans. Magn., vol. 41, no. 5, pp. 1736-1739, May 2005.

[7] L. Lebensztajn and J. L. Coulomb, "TEAM workshop problem 25: A multiobjective analysis," IEEE Trans. Magn., vol. 40, no. 2, pt. 2, pp. 1402-1405, Mar. 2004.

[8] M. C. Costa, J. L. Coloumb, and Y. Maréchal, "Parameters screening of TEAM workshop problem 25 by the application of experimental design method," in Proc. ISTET, Linz, Austria, Aug. 19-22, 2001 [Online]. Available: http://hal.archives-ouvertes.fr/hal-00173497/fr/

[9] R. Storn and K. Price, "Differential evolution-A simple and efficient heuristic for global optimization over continuous spaces," J. Global Optimiz., vol. 11, pp. 341-359, 1997. 